\title{
Multiplicative Character Sums with Counter-Dependent Nonlinear Congruential Pseudorandom Number Generators
}

\author{
Domingo Gomez * \\ Faculty of Sciences \\ University of Cantabria \\ Avd. Los Castros, Santander, Spain. \\ domingo.gomez@unican.es
}

\begin{abstract}
Nonlinear congruential pseudorandom number generators can have unexpectedly short periods. Shamir and Tsaban introduced the class of counter-dependent generators which admit much longer periods. In this paper we present a bound for multiplicative character sums for nonlinear sequences generated by counter-dependent generators.
\end{abstract}

\section{Introduction}

Let $q=p^{r}$, where $p$ is a prime number. In this paper we study a multiplicative character sum related with the distribution properties of the powers and primitive elements of counter-dependent nonlinear congruential pseudorandom number generators. This class of generators was introduced by [16] and it is defined by a recurrence of the form

$$
u_{n+1}=f\left(u_{n}, n\right), \quad u_{n} \in \mathbb{F}_{q}, \quad n=0,1, \ldots,
$$

with some initial value $u_{0}$, where $f(X, Y) \in \mathbb{F}_{q}[X, Y]$ is a polynomial over the field $\mathbb{F}_{q}$ of $q$ elements of local degree in $X$ at least 2 . It is well-known that the problem of studying the distribution of primitive roots and powers can be reduced to bound a multiplicative character sum, see, for example [9].

It is obvious that the sequence (1) eventually becomes periodic with some period $t \leq q p$. Throughout this paper we assume that this sequence is purely periodic, that is, $u_{n}=u_{n+t}$ beginning with $n=0$, otherwise we consider a shift of the original sequence.

The case $f(X, Y)=h(X) \in \mathbb{F}_{q}[X]$, which does not depend on the second variable, is the well-studied nonlinear congruential pseudorandom number generators, see $[5,8,11]$, the surveys $[18,19]$ and references therein. A bound in the corresponding multiplicative character sum was given in [10]. On the other hand,

\footnotetext{
* This work was partially supported by the Spanish Government. Research Grant
} MTM 2004-07086. Also, I want to thank A. W. for his time and patience. 
these generators have their own limits, for example the period $t$ is at most $q$. So, it is interesting to study more general pseudorandom number generators.

The counter-assisted nonlinear congruential pseudorandom number generators were defined in [16]. They are defined by the following linear recurrence:

$$
u_{n+1}=h\left(u_{n}\right)+n \quad(\bmod p) \quad 0 \leq u_{n} \leq p-1, \quad n=0,1, \ldots,
$$

where $h(X) \in \mathbb{F}_{p}[X]$. For this specific class, the linear complexity and exponential sums were studied in [2]. These generators are related to nonlinear congruential pseudorandom number generators of order 2 defined by

$$
u_{n+2}=f\left(u_{n+1}, u_{n}\right) \quad(\bmod p), \quad 0 \leq u_{n} \leq p-1, \quad n=0,1, \ldots
$$

Nonlinear congruential pseudorandom number generators of order $m \geq 2$ have been analyzed in [3,4] in particular cases and solve for the general case in [13]. The results in these papers treat the distribution of values, not distribution of powers. The linear complexity was studied in [17], so this shows that the problem is not trivial at all.

A general class of pseudorandom number generators of higher orders has been studied in $[12,14]$. This class has attracted a lot of attention, however to get a bound on the corresponding multiplicative character sum can only be done under certain conditions, see [15].

\section{Definitions and Auxiliary Results}

All the needed results are adapted, but the general properties of resultants and their proofs can be found in [1]. We use the classical abbreviation of $\operatorname{deg}_{X}$ to refer to the degree of a polynomial in the variable $X$.

The resultant is a classical concept that arises from commutative algebra. We suppose that we are working in $\mathbb{K}[X, Y]$, the ring of bivariate polynomials with coefficients in a field $\mathbb{K}$. Given two polynomials $f(X, Y), g(X, Y) \in \mathbb{K}[X, Y]$, where

$$
f(X, Y)=\sum_{i=0}^{d_{1}} f_{i}(Y) X^{i}, \quad g(X, Y)=\sum_{i=0}^{d_{2}} g_{i}(Y) X^{i} .
$$

the Sylvester matrix respect the variable $X$ is

$$
\left(\begin{array}{cccccccc}
f_{0}(Y) & f_{1}(Y) & \ldots & f_{d_{1}}(Y) & 0 & \ldots & 0 & 0 \\
0 & f_{0}(Y) & f_{1}(Y) & \ldots & f_{d_{1}}(Y) & 0 & \ldots & 0 \\
\ldots & \ldots & \ldots & \ldots & \ldots & \ldots & \ldots & \ldots \\
0 & 0 & 0 & \ldots & f_{0}(Y) & \ldots & f_{d_{1}-1}(Y) & f_{d_{1}}(Y) \\
g_{0}(Y) & g_{1}(Y) & \ldots & g_{d_{2}}(Y) & 0 & \ldots & 0 & 0 \\
0 & g_{0}(Y) & g_{1}(Y) & \ldots & g_{d_{2}}(Y) & 0 & \ldots & 0 \\
\ldots & \ldots & \ldots & \ldots & \ldots & \ldots & \ldots & \ldots \\
0 & 0 & 0 & \ldots & g_{0}(Y) & \ldots & g_{d_{2}-1}(Y) & g_{d_{2}}(Y)
\end{array}\right) .
$$


This matrix is a $\left(d_{1}+d_{2}\right) \times\left(d_{1}+d_{2}\right)$ matrix, the first row is the coefficients of $f(X, Y)$ depending on $\mathrm{Y}$, adding zeros to fill the $\left(d_{1}+d_{2}\right)$ positions. Notice that the next $d_{2}-1$ rows are shifts of the first row. The other rows are built using the polynomial $g(X, Y)$.

The determinant of this matrix is known as the resultant of the polynomials $\mathrm{f}$ and $g$ respect of the variable $X$. We will denote it by $\operatorname{Res}_{X}(f(X, Y), g(X, Y))$. The following Lemma shows the relation between resultant and common factors. It is a Corollary of [1, Proposition 1, Section 3.6].

Lemma 1 Given $f(X, Y), g(X, Y) \in \mathbb{F}_{q}[X, Y]$ then

$$
\operatorname{deg}_{X}(\operatorname{gcd}(f(X, Y), g(X, Y))) \geq 1
$$

if and only if

$$
\operatorname{Res}_{X}(f(X, Y), g(X, Y))=0 .
$$

In [6, Corollary 5.1], the author presented a relation between the composition of polynomials and resultants. His result is very general, so here is an adapted version for the proofs.

Lemma 2 Let $f(X, Y), g(X, Y), h(X, Y) \in \mathbb{K}[X, Y]$ be polynomials such as $\operatorname{deg}_{X}(f(X, Y)), \operatorname{deg}_{X}(g(X, Y)), \operatorname{deg}_{X}(h(X, Y)) \geq 1$ then,

$$
\operatorname{Res}_{X}(f(h(X, Y), Y), g(h(X, Y), Y))=\operatorname{Res}_{X}(f(X, Y), g(X, Y))^{\operatorname{deg}_{X}(h(X, Y))} .
$$

The next Lemma is a weaker version of the Bezout Theorem.

Lemma 3 Let $f(X, Y), g(X, Y) \in \mathbb{K}[X, Y]$, with $\operatorname{gcd}(f(X, Y), g(X, Y))=1$ then the number of common roots is at most the product of the degrees of the polynomials.

For a polynomial $f(X, Y) \in \mathbb{F}_{q}[X, Y]$ of total degree $d$ we define the sequence of polynomials $f_{k}(X, Y) \in \mathbb{F}_{q}[X, Y]$ by the recurrence relation

$$
f_{k+1}(X, Y)=f_{k}(f(X, Y), Y+1), \quad k=0,1, \ldots,
$$

where $f_{0}(X, Y)=X$. It is clear that $\operatorname{deg}\left(f_{k}(X, Y)\right) \leq d^{k}$ and for the sequence define in (1) that

$$
u_{n+k}=f_{k}\left(u_{n}, n\right) .
$$

The following property will be necessary in the proof of the main theorem:

Lemma 4 Given the sequence $f_{k}(X, Y) \in \mathbb{F}_{q}[X, Y]$ defined in (2) and if

$$
\operatorname{deg}_{X}\left(\operatorname{gcd}\left(f_{k}(X, Y), f_{l}(X, Y)\right)\right) \geq 1
$$

then

$$
\operatorname{deg}_{X}\left(\operatorname{gcd}\left(f_{k-i}(X, Y), f_{l-i}(X, Y)\right)\right) \geq 1, \quad \forall i \leq \min (k, l)
$$


Proof. Now, we regard the polynomials $f_{k}(X, Y), f_{l}(X, Y)$ as polynomials in the variable $X$ whose coefficients are in the ring $\mathbb{F}_{q}[Y]$ and let

$$
H(Y)=\operatorname{Res}_{X}\left(f_{k-1}(X, Y), f_{l-1}(X, Y)\right) .
$$

Using simple properties of the Sylvester Matrix, we have:

$$
\operatorname{Res}_{X}\left(f_{k-1}(X, Y+1), f_{l-1}(X, Y+1)\right)=H(Y+1)
$$

and, using Lemma 2, we get that:

$$
\operatorname{Res}_{X}\left(f_{k-1}(f(X, Y), Y+1), f_{l-1}(f(X, Y), Y+1)=H(Y+1)^{\operatorname{deg}_{X}(f(X, Y))} .\right.
$$

Applying the Lemma 1,

$$
H(Y+1)^{\operatorname{deg}_{X}(f(X, Y))}=\operatorname{Res}_{X}\left(f_{k}(X, Y), f_{l}(X, Y)\right)=0 .
$$

This clearly implies that $H(Y)=0$, therefore, again by Lemma 1 we get

$$
\operatorname{gcd}\left(f_{k-1}(X, Y), f_{l-1}(X, Y)\right)=H_{1}(X, Y), \operatorname{deg}_{X}\left(H_{1}(X, Y)\right) \geq 1 .
$$

Applying the same argument i times, we get the result.

Now, we are going to introduce some notation. Let $\chi$ be a nontrivial multiplicative character of $\mathbb{F}_{q}$, with the standard convention $\chi(0)=0$. We want to prove an upper bound on this character sum

$$
S_{\chi}(N)=\sum_{n=0}^{N-1} \chi\left(u_{n}\right)
$$

Next, we recall the classical Weil bound on multiplicative character sums (see [7, Chapter 5]) for univariate polynomials.

Lemma 5 Let $\chi$ be a character of $\mathbb{F}_{q}$ of order $s$ and let $F(X) \in \mathbb{F}_{q}[X]$ be a polynomial of positive degree that is not, up to a multiplicative constant, an sth power of a polynomial. Let $d$ be a bound on the number of distinct roots in its splitting field over $\mathbb{F}_{q}$. Under these conditions, the following inequality

$$
\left|\sum_{x \in \mathbb{F}_{q}} \chi(F(x))\right| \leq d q^{1 / 2}
$$

holds.

With this Lemma we can prove another result that we will use through later.

Lemma 6 Let $\chi$ be a character of $\mathbb{F}_{q}$ of order $s$ and let $F(X, Y) \in \mathbb{F}_{q}[X, Y]$ be a polynomial of positive degree such that $F(X, Y)$ is not, up to a multiplicative constant, an sth power of a polynomial. Let $F(X, Y)=F_{1}(X, Y)^{d_{1}} \cdots F_{h}(X, Y)^{d_{h}}$ 
the decomposition of the polynomial in a product of irreducible polynomials. Let $D$ be a bound on the total degree of $F_{1}(X, Y) \cdots F_{h}(X, Y)$. Under these conditions, the following inequality holds

$$
\left|\sum_{x, y \in \mathbb{F}_{q}} \chi(F(x, y))\right| \leq 2 D q^{3 / 2}
$$

Proof. This Lemma is trivial when $2 D \geq q^{1 / 2}$ so suppose that $2 D \leq q^{1 / 2}$. Without loss of generality, $d_{1}$ is not an integer multiple of s, because $F(X, Y)$ is not an sth power of a polynomial up to a multiplicative constant. Next,

$$
\left|\sum_{x, y \in \mathbb{F}_{q}} \chi\left(F_{1}(x, y)^{d_{1}} \ldots F_{h}(x, y)^{d_{h}}\right)\right| \leq \sum_{y \in \mathbb{F}_{q}}\left|\sum_{x \in \mathbb{F}_{q}} \chi\left(F_{1}(x, y)^{d_{1}} \ldots F_{h}(x, y)^{d_{h}}\right)\right| \text {. }
$$

Our aim is to apply Lemma 5 to each of the sums for $y$ fixed. We have to count how many times we can not apply Lemma 5 . The special cases are:

- When the polynomial $F(X, y)$ is a constant polynomial.

- When the polynomial $F(X, y)$ is an sth power.

There are, at most, $D$ different values $y$ where the polynomial $F(X, y)$ could be a constant polynomial.

Now, we consider in which cases the polynomial $F(X, y)$ is an sth power of a polynomial and how these cases will be counted.

First of all, we remark that $F(X, Y)$ is not an sth power of a polynomial, so if $F(X, y)$ is an sth power of a polynomial then we have this two possible nonexclusive situations:

- $F_{1}(X, y)^{d_{1}}$ is an $s$ th power, so because $d_{1}$ is not an $s$ multiple then we must have that $F_{1}(X, b)$ has, at least, one multiple root. This is only possible if $F_{1}(X, b)$ and the first derivative of the polynomial have a common root. $F_{1}(X, Y)$ is an irreducible polynomial, so Lemma 3 applies. We remark that the first derivative is a nonzero polynomial. Otherwise $F_{1}(X, Y)$ is a power of a polynomial, thus reducible. This can only happen in $\operatorname{deg}_{X}\left(F_{1}\right)\left(\operatorname{deg}_{X}\left(F_{1}\right)-\right.$ 1) cases.

- $F_{1}(X, b)$ and $F_{s}(X, b)$ have a common root and, by the same argument, there are at $\operatorname{most} \operatorname{deg}_{X}\left(F_{1}\right) \operatorname{deg}_{X}\left(F_{s}\right)$ possible values where it happens.

So, for each value of $y \in \mathbb{F}_{q}$, we apply Lemma 6 if the two previous cases do not occur. In the other cases, we apply the trivial bound,

$$
\begin{aligned}
\sum_{y \in \mathbb{F}_{q}}\left|\sum_{x \in \mathbb{F}_{q}} \chi(F(x, y))\right| & \leq D q+q \operatorname{deg}_{X}\left(F_{1}\right) \sum_{i=1}^{h} \operatorname{deg}_{X}\left(F_{i}\right)+D q^{3 / 2} \\
& \leq\left(D^{2}+D\right) q+D q^{3 / 2} \leq 2 D q^{3 / 2} .
\end{aligned}
$$


The last inequality holds because $2 \leq 2 D \leq q^{1 / 2}$ and this remark finishes the proof.

We call the sequence $\left(v_{n}\right)$, given by $(1)$ with $v_{0}=0$. Note that under the assumption that $\left(u_{n}\right)$ is purely periodic, the sequence $\left(v_{n}\right)$ need not be purely periodic. Let $t_{0}$ be the least period of the sequence $\left(v_{n}\right)$ if it is purely periodic and put $t_{0}=\infty$ otherwise. We are ready to prove the principal theorem:

Theorem 7. Let the sequence $\left(u_{n}\right)$, given by $(1)$ with a polynomial $f(X, Y)$ with coefficients in $\mathbb{F}_{q}[X, Y]$ and total degree $d \geq 2$ be purely periodic with period $t$ and $t \geq N \geq 1$. If $f_{k}(X, Y), 1 \leq k \leq\lceil 0.4(\log q) / \log d\rceil$ is not, up to a multiplicative constant, an sth power of a polynomial, then the bound

$$
S_{\chi}(N)=O\left(N^{1 / 2} q\left(\min \left(\frac{\log q}{\log d}, t_{0}\right)\right)^{-1 / 2}\right)
$$

holds, where the implied constant is absolute.

Proof. We can suppose that $q \geq 3$. For any integer $k \geq 0$ we have

$$
\left|S_{\chi}(N)-\sum_{n=0}^{N-1} \chi\left(u_{n+k}\right)\right| \leq 2 k
$$

so for any $K \geq 1$ and summing over $k=0,1 \ldots, K-1$, we get

$$
K\left|S_{\chi}(N)\right| \leq W+\left|\sum_{k=0}^{K-1}\left(S_{\chi}(N)-\sum_{n=0}^{N-1} \chi\left(u_{n+k}\right)\right)\right| \leq W+K^{2}
$$

where

$$
W=\sum_{n=0}^{N-1}\left|\sum_{k=0}^{K-1} \chi\left(u_{n+k}\right)\right| .
$$

By the Cauchy-Schwarz inequality and (3) we obtain

$$
\begin{array}{r}
W^{2} \leq N \sum_{n=0}^{N-1}\left|\sum_{k=0}^{K-1} \chi\left(u_{n+k}\right)\right|^{2}=N \sum_{n=0}^{N-1}\left|\sum_{k=0}^{K-1} \chi\left(f_{k}\left(u_{n}, n\right)\right)\right|^{2} \\
\leq N \sum_{x, y \in \mathbb{F}_{q}}\left|\sum_{k=0}^{K-1} \chi\left(f_{k}\left(u_{n}, n\right)\right)\right|^{2} \leq N \sum_{k, l=0}^{K-1}\left|\sum_{x, y \in \mathbb{F}_{q}} \chi\left(f_{k}(x, y)\right) \bar{\chi}\left(f_{l}(x, y)\right)\right|^{\prime}
\end{array}
$$

where $\bar{\chi}\left(f_{l}(x, y)\right)$ denotes the conjugate of $\chi\left(f_{l}(x, y)\right)$.

Because $\chi$ is a multiplicative character it is trivial to see that $\chi\left(a^{q-2}\right)=\bar{\chi}(a), \forall a \in$ $\mathbb{F}_{q}$. 
Substituting the conjugates, we get the following inequality:

$$
W^{2} \leq N \sum_{k, l=0}^{K-1}\left|\sum_{x, y \in \mathbb{F}_{q}} \chi\left(f_{k}(x, y) f_{l}(x, y)^{q-2}\right)\right| .
$$

Next we have to show that for $0 \leq l \leq k \leq K-1$ the polynomial $F(X, Y)=$ $f_{k}(X, Y) f_{l}(X, Y)^{q-2}, k \geq l$ is, up to a multiplicative constant, an $s$ th power of a polynomial only if $k \equiv l \bmod t_{0}$, where $k \equiv l \bmod \infty$ means $k=l$.

Suppose $g(X, Y)=\operatorname{gcd}\left(f_{k}(X, Y), f_{l}(X, Y)\right)$ has degree at least 1 in $X$. By Lemma $4, \operatorname{gcd}\left(f_{0}(X, Y)=X, f_{k-l}(X, Y)\right)$ is a non constant polynomial in $X$. Because $X$ is a prime polynomial, we have that the greatest common divisor between $f_{0}(X, Y)$ and $f_{k-l}(X, Y)$ is $X$ so $v_{k-l}=0$ and, consequently, $k-l \equiv 0 \bmod t_{0}$.

Now suppose $k \not \equiv l \bmod t_{0}$ and thus $g(X, Y)=1$. Hence, if $F(X, Y)$ is (up to a multiplicative constant) an sth power, then both $f_{k}(X, Y)$ and $f_{l}(X, Y)$ are (up to multiplicative constants) sth powers, which is a contradiction to our assumption provided that $K$ is small enough (this will be guaranteed by the subsequent choice of $K)$. Now the number of pairs $(k, l) \in \mathbb{Z}^{2}$ with $0 \leq l<k \leq K-1$ and $k \equiv l \bmod t_{0}$ is at most $K^{2} /\left(2 t_{0}\right)$. For these pairs $(k, l)$ we estimate the inner sum in the last bound on $W^{2}$ trivially by $q$. For all other pairs we can use Lemma 6 and get

$$
W^{2}<K N q^{2}+K^{2} N\left(\frac{q^{2}}{t_{0}}+2 d^{K-1} q^{3 / 2}\right) .
$$

With

$$
K:=\left\lceil 0.4 \frac{\log q}{\log d}\right\rceil
$$

we get the result and this finishes the proof.

\section{References}

1. David Cox, John Little, and Donal O'Shea. Ideals, varieties, and algorithms. Undergraduate Texts in Mathematics. Springer, New York, third edition, 2007. An introduction to computational algebraic geometry and commutative algebra.

2. Edwin El-Mahassni and Arne Winterhof. On the distribution and linear complexity of counter-dependent nonlinear congruential pseudorandom number generators. Journal of Algebra, Number Theory and Applications, 2:1-6, 2006.

3. Frances Griffin, Harald Niederreiter, and Igor E. Shparlinski. On the distribution of nonlinear recursive congruential pseudorandom numbers of higher orders. In $A p$ plied algebra, algebraic algorithms and error-correcting codes (Honolulu, HI, 1999), volume 1719 of Lecture Notes in Comput. Sci., pages 87-93. Springer, Berlin, 1999.

4. Jaime Gutierrez and Domingo Gomez-Perez. Iterations of multivariate polynomials and discrepancy of pseudorandom numbers. In Applied algebra, algebraic algorithms and error-correcting codes (Melbourne, 2001), volume 2227 of Lecture Notes in Comput. Sci., pages 192-199. Springer, Berlin, 2001. 
5. Jaime Gutierrez, Igor E. Shparlinski, and Arne Winterhof. On the linear and nonlinear complexity profile of nonlinear pseudorandom number-generators. IEEE Trans. Inform. Theory, 49(1):60-64, 2003.

6. Hoon Hong. Subresultants under composition. J. Symbolic Comput., 23(4):355365, 1997.

7. Rudolf Lidl and Harald Niederreiter. Finite fields, volume 20 of Encyclopedia of Mathematics and its Applications. Cambridge University Press, Cambridge, second edition, 1997. With a foreword by P. M. Cohn.

8. Harald Niederreiter and Igor E. Shparlinski. On the distribution and lattice structure of nonlinear congruential pseudorandom numbers. Finite Fields Appl., 5(3):246-253, 1999.

9. Harald Niederreiter and Igor E. Shparlinski. On the distribution of power residues and primitive elements in some nonlinear recurring sequences. Bull. London Math. Soc., 35(4):522-528, 2003.

10. Harald Niederreiter and Arne Winterhof. Multiplicative character sums for nonlinear recurring sequences. Acta Arith., 111(3):299-305, 2004.

11. Harald Niederreiter and Arne Winterhof. Exponential sums for nonlinear recurring sequences. Finite Fields Appl., 14(1):59-64, 2008.

12. Alina Ostafe. Multivariate permutation polynomial systems and nonlinear pseudorandom number generators. Finite Fields and Their Applications, 16(3):144-154, 2010 .

13. Alina Ostafe, Elena Pelican, and Igor E. Shparlinski. On pseudorandom numbers from multivariate polynomial systems. Finite Fields and their Applications (to appear), 2010.

14. Alina Ostafe and Igor E. Shparlinski. On the degree growth in some polynomial dynamical systems and nonlinear pseudorandom number generators. Math. Comp., 79(269):501-511, 2010.

15. Alina Ostafe, Igor E. Shparlinski, and Arne Winterhof. Multiplicative character sums of a class of nonlinear recurrence vector sequences. Preprint, 2010.

16. Adi Shamir and Boaz Tsaban. Guaranteeing the diversity of number generators. Inform. and Comput., 171(2):350-363, 2001.

17. Alev Topuzoğlu and Arne Winterhof. On the linear complexity profile of nonlinear congruential pseudorandom number generators of higher orders. Appl. Algebra Engrg. Comm. Comput., 16(4):219-228, 2005.

18. Alev Topuzoğlu and Arne Winterhof. Pseudorandom sequences. In Topics in geometry, coding theory and cryptography, volume 6 of Algebr. Appl., pages 135166. Springer, Dordrecht, 2007.

19. Arne Winterhof. Recent results on recursive nonlinear pseudorandom number generators. In Sequences and their Applications SETA 2010, Lecture Notes in Comput. Sci. Springer, 2010. 